\title{
Optimization and Predictive Control of a Vapor Compression Cycle under Transient Pulse Heat Load
}

\author{
Zehao Yang ${ }^{\mathrm{a}, *}$, Daniel T. Pollock ${ }^{\mathrm{a}}$, John T. Wen ${ }^{\mathrm{b}}$ \\ ${ }^{a}$ Department of Mechanical, Aerospace and Nuclear Engineering \\ ${ }^{b}$ Department of Industrial and System Engineering, \\ Rensselaer Polytechnic Institute, 110 8th Street, Troy, NY 12180, USA
}

\begin{abstract}
There are well established control methods to stabilize the vapor compression cycle (VCC) about a given operating point. However, it is challenging to design such local controllers to handle large transient heat flux disturbances, due to complex coupling and constraints, and potential violation of critical heat flux (CHF) which could lead to the damaging dryout condition. Since VCC's are locally stable with relatively slow dynamics, model predictive control (MPC) is ideally suited to address these challenges. MPC solves a constrained receding horizon minimization problem under known transient heat disturbance. The objective function is a combination of the exit evaporator wall temperature, which provides an indication for the onset of partial dryout, energy consumption, and control input effort. This paper presents results from the application of MPC to a VCC testbed in our laboratory. We show that MPC can significantly increase the robustness with respect to transient disturbances by moving the system to an advantageous operating point in anticipation of known disturbances.
\end{abstract}

Keywords: model predictive control, electronics cooling, vapor compression cycle, critical heat flux

${ }^{*}$ Corresponding author: Zehao Yang; Tel.: +1-518-276-2124; fax: +1-518-276-4897 E-mail: yangz5@rpi.edu (Z. Yang)

Preprint submitted to International Journal of Refrigeration

December 15, 2016

(C) 2017. This manuscript version is made available under the Elsevier user license http://www.elsevier.com/open-access/userlicense/1.0/ 


\begin{tabular}{|c|c|}
\hline Nomenclature & plant output \\
\hline$A, B, B_{0}, C, D$ state-space matrices & Greek symbols \\
\hline valve area opening $[\%]$ & compressor volumetric effi- \\
\hline hydraulic diameter $[\mathrm{m}]$ & ciency \\
\hline nonlinear model function & density $\left[\mathrm{kg} \mathrm{m}^{-3}\right]$ \\
\hline sampling frequency $[\mathrm{Hz}]$ & compressor speed $[\mathrm{Hz}]$ \\
\hline objective function & \\
\hline controller gain & Superscript \\
\hline discrete time step & steady-state value \\
\hline $\begin{array}{ll}k_{v} \quad \text { valve discharge coefficient } \\
{\left[\mathrm{m}^{2}\right]}\end{array}$ & Subscripts \\
\hline mass flow rate $\left[\mathrm{kg} \mathrm{s}^{-1}\right]$ & accumulator \\
\hline discrete prediction horizon & actual \\
\hline $\begin{array}{ll}N_{u} & \text { number of measurement } \\
& \text { samples within a control in- } \\
& \text { terval }\end{array}$ & $\begin{array}{ll}c & \text { condenser } \\
\text { crit } & \text { critical } \\
d & \text { discrete time }\end{array}$ \\
\hline $\begin{array}{ll}\Delta P & \text { pressure difference across the } \\
& \text { valve }[\mathrm{Pa}]\end{array}$ & $\begin{array}{l}\text { evaporator } \\
\text { end }\end{array}$ \\
\hline pressure $[\mathrm{Pa}]$ & integral gain \\
\hline diagonal weighting matrix & compressor \\
\hline heating power $[\mathrm{W}]$ & energy \\
\hline temperature $[\mathrm{K}]$ & outlet \\
\hline time $[\mathrm{s}]$ & proportional gain \\
\hline sampling period $[\mathrm{s}]$ & setpoint/reference \\
\hline control inputs: $\dot{m}_{v}, \omega, q_{a}$ & temperature \\
\hline actuator inputs: $A_{v}, \omega, q_{a}$ & control inputs \\
\hline work $[J]$ & wall \\
\hline system state vector & valve \\
\hline vapor quality & \\
\hline
\end{tabular}

\section{Introduction}

Electronic devices are designed and manufactured with higher power density to meet the rising demand for faster computing, larger storage, higher 
bandwidth communication and smaller footprint for portability. The increase in power density leads to the concomitant rise in heat which must be efficiently removed to maintain performance. The most common approach is single-phase cooling, such as air cooling. Due to the low heat transfer coefficient of air, single-phase cooling applied to high heat flux devices usually results in high power consumption and low cooling efficiency. As power density continues to rise, there is an urgent need for new cooling solutions with higher cooling capability and efficiency.

Two-phase cooling is a promising candidate to meet these challenges as it can potentially provide a significantly higher rate of heat transfer. However, cooling cycles that implement two-phase flow, such as the vapor compression cycles (VCC's) and pumped-liquid refrigeration systems, have complex input and state constraints, system couplings, and nonlinearity, posing challenges for control over wide operating conditions. The control of traditional VCC's such as heating, ventilation and air-conditioning (HVAC) and refrigeration systems typically focuses on temperature tracking and energy efficiency $[1,2,3,4]$. For electronics cooling involves imposed heat flux, a major additional consideration is the violation of the critical heat flux $(\mathrm{CHF})$ condition. When the input heat flux exceeds the CHF, there could be dryout or transition to film boiling, resulting in a sudden drop of the heat transfer coefficient, and a rapid and dangerous rise of the wall temperature. The typical approach to addressing $\mathrm{CHF}$ is to operate with a large safety margin (low exit quality) and use secondary evaporators to reach superheated exit flow. $[5,6,7,8]$. Such approach ensures CHF avoidance but compromises efficiency. Our own group has been using model based disturbance rejection control combined with gain scheduling to address large transient heat load $[9,10,11]$. In VCC control design, constraints are typically not directly addressed. Instead, controller gains are chosen to avoid the constraint under the expected operating conditions. For the input constraints, the usual strategy is saturation with integral anti-windup $[12,11]$.

Model predictive control (MPC) has long been used in process control [13, 14]. MPC solves a finite horizon optimal control problem based on the process model and imposed state and input constraints. After the implementation of the first input step, the optimization is repeated with the latest measurements as the initial condition. A key advantage of MPC is its ability to handle input and state constraints as they are built into the optimization problem and the feedback action adding robustness to noise and modeling error. MPC has been applied to traditional VCC systems with the focus primarily on 
evaporator temperature regulation and efficiency enhancement $[15,16,17,18$, 19]. Control objectives typically include penalty on temperature deviation, power consumption, and a terminal cost (to ensure stability). The CHF avoidance has not been explicitly considered. The choice of the prediction horizon can significantly affect the MPC performance. Longer prediction horizon may result in better controller performance at the cost of higher computation load and larger accumulated error (due to model uncertainty) at the end of the prediction horizon. For MPC applied to traditional VCC systems, the prediction horizon varies from 20 seconds [20] to 24 hours [16], depending on the application.

This paper considers electronics cooling with known heat flux disturbance input. In applications such as high-power radar systems, electromagnetic weaponry and server farms, the load pattern may be known a priori, either through a prior schedule or past usage patterns. MPC is ideally suited for these scenarios - when the look-ahead horizon overlaps with the transient heat flux input, the algorithm would prepare for this transient load before its actual occurrence. The main objective of the paper is CHF avoidance during these known heat load disturbances. Nonlinear simulation and experimental validation are used to tune the controller parameters and validate the controller design on a single-evaporator VCC testbed. For each static heat load, the operating point condition is chosen based on a static optimization balancing safety margin (in terms of the distance from CHF) and efficiency (in terms of the coefficient of performance, COP) [21, 9]. Our approach uses an inner/outer loop control architecture around a chosen operating point. The inner loop maintains a given mass flow rate setpoint, while the outer loop adjusts the setpoint based on the MPC solution. Since the evaporator operates entirely in the two-phase regime, a simple one-zone reduced order model is used for the optimal control solution in MPC. This work contributes to the arsenal of control methodologies for VCC operation; in particular, it is the first application of MPC to VCC under high transient heat flux with the focus on $\mathrm{CHF}$ avoidance.

The structure of this paper is organized as follows: Section 2 introduces the VCC testbed and the nonlinear model. Section 3 formulates the control problem including the two-loop architecture, and the objective functions and constraints in the optimization problem. Section 4 presents the nonlinear simulation result and guideline for choosing the prediction horizon based on the disturbance magnitude. Section 5 shows the experimental results with MPC applied to disturbance pulses of various size and duration as well as a 


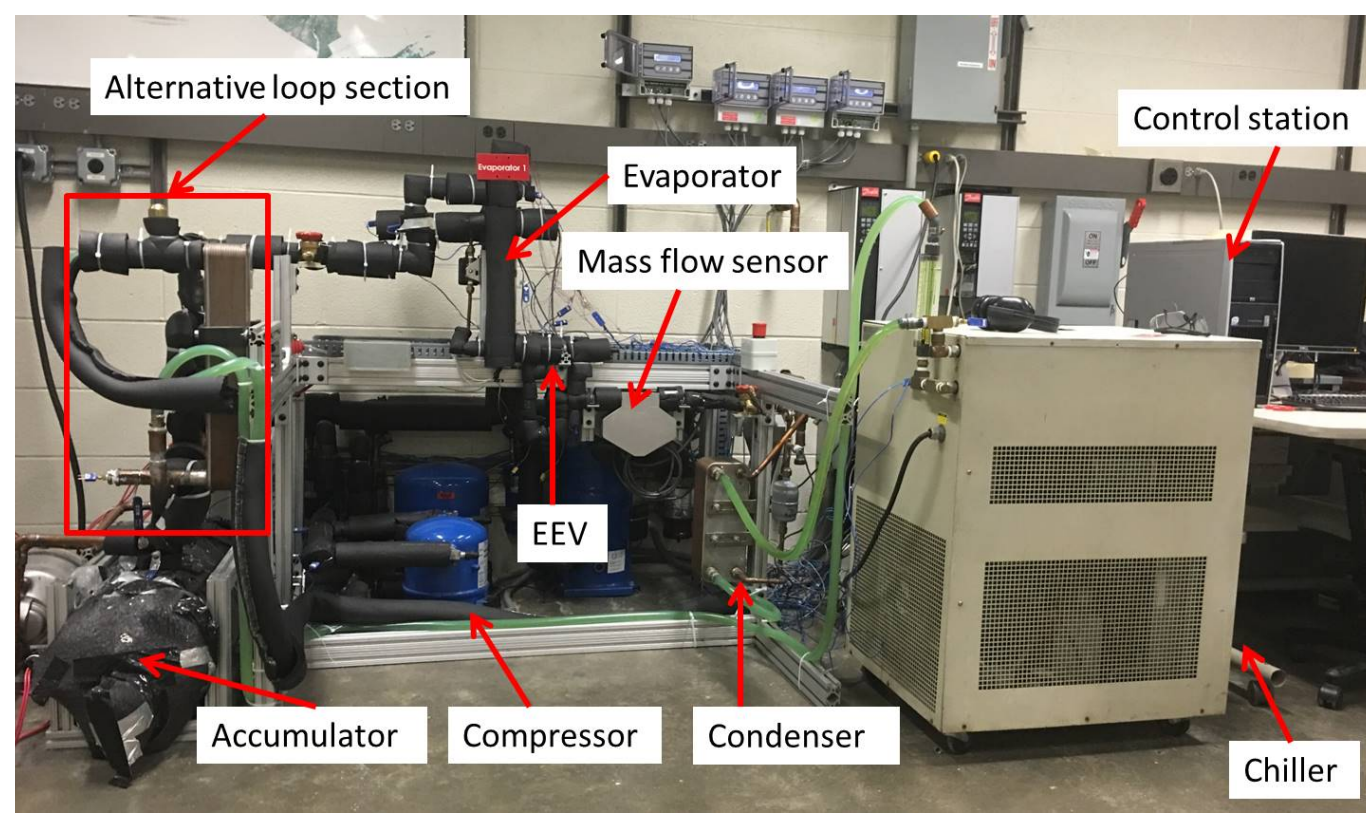

Fig. 1: VCC testbed

pulse train.

\section{System Description}

\subsection{Experimental Testbed}

The VCC system discussed in this paper is comprised of five main components: a condenser, an electronic expansion valve (EEV), an evaporator, a heated accumulator, and a compressor. A chiller is implemented to fix the temperature of the cooling water. The refrigerant used in this testbed cooling cycle is R134a. Fig. 1 shows the layout of the VCC testbed. Measurements are collected from key locations in the VCC (shown in Fig. 2) using the National Instruments (NI) Data Acquisition (DAQ) system, and the VCC is controlled using NI LabVIEW.

Fig. 2 shows the structure of the VCC loop. The compressor forces vapor refrigerant to a high-pressure high-temperature state, and releases it into the discharge line. The superheated refrigerant then passes through the plate condenser where heat exchange occurs with a secondary cooling water loop. The refrigerant leaves the condenser subcooled near a fixed cooling water temperature due to the high water flow rate and large heat exchanger 


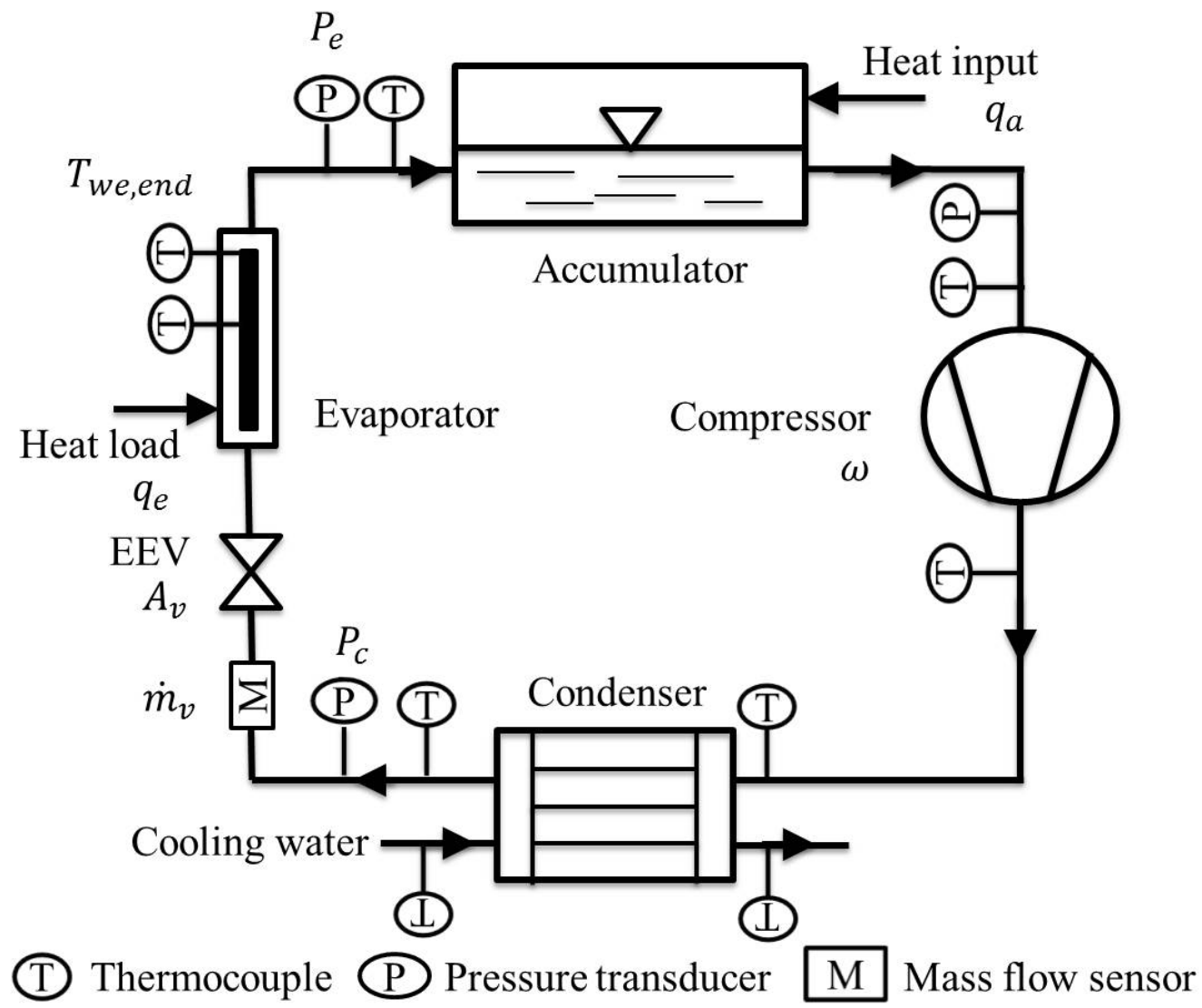

Fig. 2: VCC layout

size. The high-pressure subcooled refrigerant is then discharged through the EEV into the evaporator as a low-quality two-phase mixture. Inside the evaporator, the refrigerant passes through the vertical annular channel around the cartridge heater where heat is removed and carried out of the evaporator. The two-phase refrigerant, at high vapor quality, flows into the accumulator where its liquid and vapor contents are separated. The vapor content of the refrigerant is allowed back to the compressor while some of the liquid content is evaporated via external electric heaters to stabilize the liquid level.

Absolute pressure transducers are placed at the exit of the condenser and after the evaporator outlet to measure the condenser pressure, $P_{c}$, and 
evaporator pressure, $P_{e}$. The operating range for these pressure transducers are 0 - 250 psi $(0-1724 \mathrm{kPa})$ and $0-100$ psi $(0-689 \mathrm{kPa})$, respectively. The evaporator end-point wall temperature, $T_{w e, e n d}$, is measured using a type- $T$ thermocouple soldered on the end of the cartridge heater located at the center of the evaporator. Refrigerant mass flow through the valve, $\dot{m}_{v}$, is measured using a Coriolis mass flow meter placed before the valve (ACM 300 by AW Co. [22]). The operating range of this flow meter is $0-5 \mathrm{~kg} \mathrm{~min}{ }^{-1}$ $\left(0-83 \mathrm{~g} \mathrm{~s}^{-1}\right)$. The uncertainty of the pressure transducers, thermocouples and mass flow sensors are $\pm 0.3 \%$ of the full range, $\pm 0.3{ }^{\circ} C$, and $\pm 0.5 \%$ of the measurement, respectively $[22,23,24]$. Note that we designed our testbed to use the Coriolis flow meter for its superior accuracy. However, other lower-cost industrial mass flow meters may be used as alternatives as well. Coriolis flow meter also contains a built-in digital low pass filter to remove the transient noise. Due to the thermal mass of the evaporator wall, the heat load to mass flux dynamics of our system is slow, with bandwidth less than $0.05 \mathrm{~Hz}$ [9], so the effect of this digital filter is negligible. The cooling capacity of the single-evaporator VCC is $1500 \mathrm{~W}$, determined by the maximum mass flow rate of the VCC.

\subsection{Nonlinear Model}

We use the lumped-parameter VCC model for our testbed developed by our group in [9]. Condenser, evaporator, and accumulator dynamics are modeled using a one-zone approximation of the mass and energy balance equations. The valve and compressor are treated as static components as they have much faster dynamics. The overall model is of the following affinein-input form:

$$
\dot{X}=f_{0}(X)+f_{1}(X) v+f_{2}(X) q_{e}
$$

where $X=\left[\begin{array}{llllllll}x_{c} & P_{c} & T_{w, c} & x_{e} & P_{e} & T_{w, e} & T_{w e, e n d} & T_{w, a}\end{array}\right]^{T}$ is the full state consisting of the condenser average quality, exit pressure, and average wall temperature, evaporator average quality and exit pressure, and average and endpoint wall temperatures, and accumulator wall temperature, $v=\left[\begin{array}{lll}A_{v} & \omega & q_{a}\end{array}\right]^{T}$ is the control input, and $q_{e}$ is the imposed heat flux to be removed. Functions $\left(f_{0}, f_{1}, f_{2}\right)$ are highly nonlinear and involves thermodynamic property table lookup for R134a and empirically identified heat transfer coefficients under different operating conditions. They may be found in [9] and will not be reproduced here. The output is given by the sensors instrumented on the 
testbed:

$$
y=\left[\begin{array}{c}
P_{c} \\
P_{e} \\
T_{w e, e n d}
\end{array}\right]=C X, \quad C=\left[\begin{array}{cccccccc}
0 & 1 & 0 & 0 & 0 & 0 & 0 & 0 \\
0 & 0 & 0 & 0 & 1 & 0 & 0 & 0 \\
0 & 0 & 0 & 0 & 0 & 0 & 1 & 0
\end{array}\right] .
$$

The mass flow rate, $\dot{m}_{v}$, is linearly related to the valve opening by a positive state-dependent gain as given by the orifice equation

$$
\dot{m}_{v}=k_{v} A_{v} \sqrt{2 \rho_{v}\left(P_{c}-P_{e}\right)} .
$$

Though $\dot{m}_{v}$ is measured, we do not include it in the output vector since, as will be shown in the next section, we will close an inner loop around $A_{v}$ to turn the setpoint for $\dot{m}_{v}$ into a virtual input. The mass flow rate through the compressor depends on the compressor speed, $\omega$, and is given by

$$
\dot{m}_{m}=\rho_{a} \eta_{V} V_{m} \omega
$$

where $\eta_{V}$ is the compressor volumetric efficiency, $\rho_{a}$ is the inlet vapor density, and $V_{m}$ is the stroke volume. Both $\rho_{a}$ and $\eta_{V}$ are monotonically increasing with respect to $P_{e}$. Therefore, for a fixed mass flow rate, $P_{e}$ is monotonically decreasing function of $\omega$. We shall use this fact to gain intuition on the impact of compressor speed control on the VCC operation.

We may state CHF in terms of critical quality which is the flow quality at which CHF typically occurs. A correlation for critical quality based on mass flux and pressure is used to predict onset of dryout at the evaporator outlets. For our testbed, we have obtained a critical quality correlation experimentally using a modified version of Levitan and Lantsmans correlation [9, 25]:

$$
x_{\text {crit }}=\left(0.008 / D_{h}\right)^{0.15}\left(0.113+3.48 \cdot 10^{-7} P_{e}\right)\left(\frac{1000 A_{e}}{\dot{m}_{e}}\right)^{0.5}
$$

where $D_{h}$ is the hydraulic diameter of the evaporator, $A_{e}$ is the cross-sectional area of the annular channel around the cartridge heater. For higher CHF, it is therefore advantageous to increase $P_{e}$. Higher mass flow rate has two effects on heat removal: on the positive side, it increases the average rate of heat transfer, but from the correlation above, it also lowers the CHF (at the evaporator exit). We shall see later that the solution of the optimal control problem will balance these two effects. 


\section{Control Problem Formulation}

\subsection{Two-Loop Control Architecture}

In this paper, we focus on the input disturbance heat rejection about a fixed operating point. For a given constant heat load $q_{e}^{*}$, we may solve for the input $v^{*}$ that optimizes a combination of the steady state efficiency (characterized by a high coefficient of performance, COP) and dryout avoidance (characterized by a high CHF) [21]. Let the corresponding state be $X^{*}$, i.e.,

$$
f_{0}\left(X^{*}\right)+f_{1}\left(X^{*}\right) v^{*}+f_{2}\left(X^{*}\right) q_{e}^{*}=0 .
$$

Note that our VCC system is open loop (locally) stable as we assume negligible pressure drops across the heat exchangers (which is true for macro-

scale, but not micro-scale, heat exchangers). We consider the control problem as choosing the input deviation from the steady state, $\delta v=v-v^{*}=$ $\left[\begin{array}{lll}\delta A_{v} & \delta \omega & \delta q_{a}\end{array}\right]$ to avoid undesirable conditions (such as dryout) under a known transient evaporator heat disturbance $\delta q_{e}$.

Mass flow rate directly affects the average heat transfer rate in the evaporator. It is therefore preferable to directly adjust it in the optimization, without the concern of the nonlinearity in the valve equation. In order to handle nonlinearity, Larsen [17] and Elliot [26] have implemented inner-loop control linearization approach in their MPC controller design for their vapor compression systems. To reduce the effect of the valve nonlinearity, we use an inner/outer-loop structure as shown in Fig. 3. The inner loop adjusts $A_{v}$ based on the $\delta \dot{m}_{v}$ feedback to track the specified setpoint $\delta \dot{m}_{v, s e t}$. The outer loop chooses the refrigerant mass flow setpoint $\delta \dot{m}_{v, s e t}$, compressor speed $\delta \omega$, and accumulator heat $\delta q_{a}$ to ameliorate the effect of the know disturbance input. Consider a proportional-integral (PI) controller for the valve:

$$
\delta A_{v}=-K_{P}\left(\delta \dot{m}_{v}-\delta \dot{m}_{v, s e t}\right)-K_{I} e, \quad \dot{e}=\delta \dot{m}_{v}-\delta \dot{m}_{v, \text { set }}
$$

In the valve equation (3), if we regard the state-dependent gain simply as a positive time-varying parameter, then the PI controller would drive $\dot{m}_{v}$ to $\dot{m}_{v, s e t}$ with the rate approximately $K_{I} / K_{P}$. For experiments presented in this paper, the gains are chosen to be $K_{P}=3000 \% /(\mathrm{kg} / \mathrm{s})$ and $K_{I}=$ $600 \% / \mathrm{kg}$, so the inner loop time constant is $K_{P} / K_{I}=5 \mathrm{~s}$ (note the valve opening is expressed as the percentage of the full opening). We will choose the MPC control update rate at $50 \mathrm{~s}$ and $100 \mathrm{~s}$ based on the length of the 


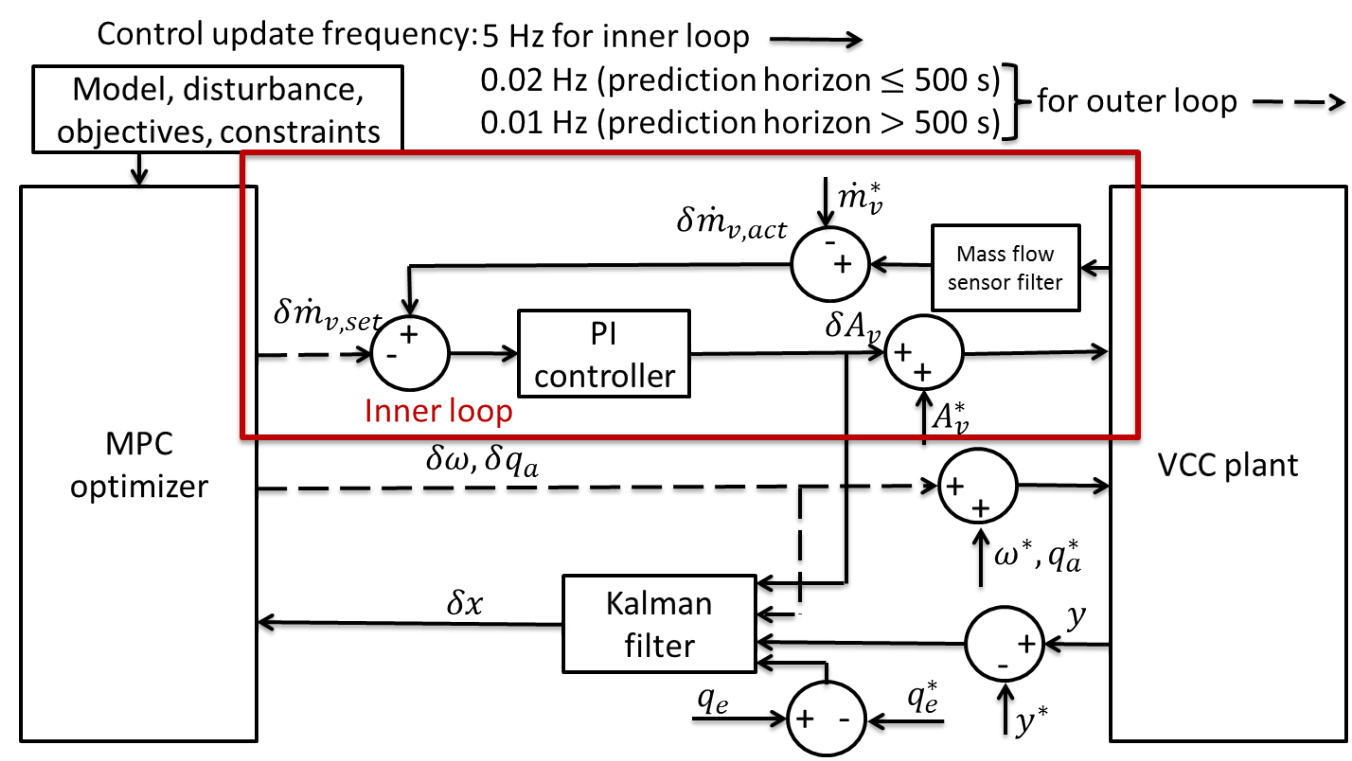

Fig. 3: Structure of the inner/outer-loop MPC. The inner-loop PI controller tracks the refrigerant mass flow rate using the EEV and the outer-loop MPC optimizes for the optimal evaporator mass flow rate, the compressor speed and the accumulator heat.

prediction horizon. This order of magnitude separation of time scale allows us to consider $\dot{m}_{v}$ as the control variable in the MPC solution.

Coriolis mass flow meters have built-in filters to reduce measurement noise. The filter used on AW Company Coriolis mass flow sensors has a default time constant of $1 \mathrm{~s}$ [27]. The measurement error due to a step change in the mass flow rate reduces to less than $3 \%$ after $3 \mathrm{~s}$. Since the mass flow setpoint from the MPC updates every $50 \mathrm{~s}$ or $100 \mathrm{~s}$ (depending on the length of the prediction horizon), the dynamics of the filter does not have a noticeable influence on the stability and performance of the inner loop.

The inner loop is introduced to reduce the effect of valve nonlinearity. Valve nonlinearity can affect the performance of the MPC especially for oversized valves and for operations away from the nominal operating condition. If the valve behavior is sufficiently linear, the inner loop can be eliminated, and the valve opening can be directly used as the optimization variable for the MPC. 


\subsection{Outer-Loop Optimal Control}

With the inner loop closed, the inputs for the outer loop control become $u=\left[\begin{array}{lll}\dot{m}_{v, \text { set }} & \omega & q_{a}\end{array}\right]^{T}$ and the state equation (1) may be modified as

$$
\dot{X}=f_{0}(X)+\bar{f}_{1}(X) u+f_{2}(X) q_{e}
$$

by eliminating $A_{v}$ using (3) and replacing $\dot{m}_{v}$ with $\dot{m}_{v, s e t}$. Denote the nominal outer loop input and state corresponding to the nominal heat load $q_{e}^{*}$ as $u^{*}$ and $X^{*}$. The system linearized about this operating point is given by

$$
\begin{aligned}
\delta \dot{X}= & \underbrace{\left(\nabla_{X} f_{0}\left(X^{*}\right)+\nabla_{X}\left(\bar{f}_{1}\left(X^{*}\right) u^{*}\right)+\nabla_{X}\left(f_{2}\left(X^{*}\right) q_{e}^{*}\right)\right)}_{A} \delta X \\
& +\underbrace{\bar{f}_{1}\left(X^{*}\right)}_{B} \delta u+\underbrace{f_{2}\left(X^{*}\right)}_{B_{0}} \delta q_{e}
\end{aligned}
$$

where $\delta X=X-X^{*}, \delta u=u-u^{*}, \delta q_{e}=q_{e}-q_{e}^{*}$, and $\nabla_{X} f_{0}\left(X^{*}\right)$ means the gradient of $f_{0}(X)$ evaluated at $X^{*}$. The linearized system is stable, since the open loop system with the inner loop closed is locally stable. The sensor measurements are acquired at the $f_{s}=5 \mathrm{~Hz}$ for our system (sampling period $\left.T_{s}=0.2 \mathrm{~s}\right)$. Write the discretized sampled-data system as

$\delta X(k+1)=A_{d} \delta X(k)+B_{d} \delta u(k)+B_{d_{0}} \delta q_{e}(k), \quad \delta y(k)=C_{d} \delta X(k), \quad k=0,1, \ldots$

where the discrete time transfer function $C_{d}\left(z I-A_{d}\right)^{-1}\left[B_{d}, B_{d_{0}}\right]$ is the Tustin transform of the continuous time transfer function $C(s I-A)^{-1}\left[B, B_{0}\right]$ with sampling frequency $f_{s}$.

To avoid excessive computation in the optimization, we update the input $\delta u$ at a slower rate by holding $\delta u$ constant for $N_{u}$ samples. Computation time increases with the prediction horizon (due to longer time needed for the model simulation) and the number of control steps (more optimization variables). In order to provide the necessary prediction length and maintain a reasonable computation time, we chose for our testbed $N_{u}=250$ (i.e. control interval of $50 \mathrm{~s}$ ) for the horizon of $500 \mathrm{~s}$ or less, and $N_{u}=500$ (i.e. control interval of $100 \mathrm{~s}$ ) for the horizon greater than $500 \mathrm{~s}$ and the frequency of control update is $0.02 \mathrm{~Hz}$ and $0.01 \mathrm{~Hz}$, respectively. It is possible to choose the length of the prediction horizon based on the size and duration of the disturbance. The outer loop optimal control problem chooses the inputs at $N_{p}$ control steps to minimize a specified objective function. The control 
horizon is then $N_{p} N_{u} T_{s}$ s. The control input trajectory consists of $N_{p}$ input vectors: $\left\{\delta u\left(j N_{u}\right), j=0, \ldots, N_{p}-1\right\}$, and the complete $\delta u$ is a staircase function:

$$
\delta u(k)=\delta u\left(j N_{u}\right) \quad \text { if } j N_{u} \leq k<(j+1) N_{u}, \quad j=0 \ldots N_{p}-1 .
$$

\subsection{Objective Function}

We pose an optimization problem to ensure the avoidance of excessively high temperature in the evaporator during the transient disturbance (based on the CHF consideration) while maintaining energy efficiency. This section discusses the design of the objective function of the optimization problem.

At each mass flow rate and evaporator pressure, there is a corresponding critical quality $x_{\text {crit }}$ for CHF [25]. The separation between the exit quality from the critical quality, $\Delta x_{c r i t}:=x_{\text {crit }}-x_{o}$, characterizes the distance from CHF. The wall heat transfer coefficient decreases as $\Delta x_{\text {crit }}$ approaches zero (see [11] for a correlation derived based on our testbed), and correspondingly, an increase of the steady state wall temperature (for each fixed $\Delta x_{\text {crit }}$ ). Hence, the evaporator wall temperature is a good indicator of the onset of CHF violation, corresponding to partial dryout. The refrigerant flow reaches the highest vapor quality, and the lowest heat transfer coefficient, at the end of the evaporator. Therefore, we use the evaporator end-point wall temperature, $T_{w e, e n d}$, as an indicator of the onset of CHF. The following single-sided penalty function of $\delta T_{w e, e n d}$

$$
J_{T}=\sum_{k=0}^{N_{p} \cdot N_{u}-1}\left(\max \left(0, \delta T_{w e, \text { end }}(k)\right)\right)^{3} .
$$

We only penalize the temperature rise in the evaporator because temperature drop or thermal energy removal from the evaporator wall prior to the disturbance can be utilized by the MPC to increase cooling potential in preparation for the incoming disturbance. The cubic function places a heavier penalty on the higher temperature regime.

A secondary control objective minimizes energy consumption away from the nominal condition. The sources consist of the compressor work, $W_{m}$, which is proportional to the compressor mass flow rate, and accumulator heat, $q_{a}$ :

$$
J_{E}=\sum_{k=0}^{N_{p} \cdot N_{u}-1}\left(W_{m}(k)-W_{m}^{*}\right)+\left(q_{a}(k)-q_{a}^{*}\right)
$$


where $W_{m}^{*}$ and $q_{a}^{*}$ are the energy input at the nominal operating condition. Energy minimization may be in conflict with CHF avoidance because refrigerant mass flow increase for added cooling capacity would require higher compressor speed (and therefore work) and accumulator heat input (to add more refrigerant to the cooling loop). When there is a large transient disturbance in the optimization horizon, the optimal control will use more energy to increase the cooling capacity in the evaporator. After the disturbance passes, the controller will return to nominal operating condition.

To ensure the optimization problem is well-posed and also to prevent large actuation effort, we also add a quadratic control input penalty to the objective function:

$$
J_{\delta u}=\sum_{j=0}^{N_{p}-1} \delta u\left(j N_{u}\right)^{T} Q \delta u\left(j N_{u}\right)
$$

where $Q$ is a diagonal weighting matrix with entries $(5 e 5,10,1 e-4)$, selected to normalize units, avoid input saturation (hard constraints will also be added) and balance with the other two objective functions. The overall objective function is the sum of the three terms described above:

$$
J=J_{T}+J_{E}+J_{\delta u}
$$

\subsection{Input and State Constraints}

The evaporator wall temperature typically has an imposed upper limit for safety and performance considerations. For example, the maximum allowable temperature for $\mathrm{CPU}$ cooling is $85-90^{\circ} \mathrm{C}[28,29]$. For this research, we set the upper bound at

$$
T_{w e, e n d} \leq 85^{\circ} \mathrm{C} .
$$

This hard constraint will prevent the predicted temperature trajectory from violating this maximum temperature, but the temperature may get close to the bound. The soft constraint (10) in the objective function will further steer the temperature trajectory a safe distance away.

The pressure transducers instrumented on the VCC testbed are rated to a maximum allowable pressure of $250 \mathrm{psi}(1724 \mathrm{kPa})$. We set the upper bound for the condenser pressure to $1250 \mathrm{kPa}$ to provide a safety margin. A minimum condenser pressure $650 \mathrm{kPa}$ is also required to ensure subcooling in the liquid line to avoid valve choking. The cooling water temperature is 
$21^{\circ} \mathrm{C}$ with the corresponding saturation pressure at about $600 \mathrm{kPa}$. If the refrigerant enters the two-phase regime below this pressure, small bubbles could form and chock the valve. The minimum evaporator pressure is set at $110 \mathrm{kPa}$ to guarantee the pressure inside the VCC is higher than atmospheric pressure to prevent external gas leak into the VCC causing component damages. To summarize, we have the following set of inequality constraints for the heat exchanger pressures:

$$
\left[\begin{array}{c}
-P_{c} \\
P_{c} \\
P_{e}
\end{array}\right] \geq\left[\begin{array}{c}
-1250 \mathrm{kPa} \\
650 \mathrm{kPa} \\
110 \mathrm{kPa}
\end{array}\right]
$$

Additional constraints are imposed due the physical limitation of the components in our setup:

$$
\left[\begin{array}{c}
0.006 \mathrm{~kg} \mathrm{~s}^{-1} \\
45 \mathrm{~Hz} \\
0 \mathrm{~W}
\end{array}\right] \leq\left[\begin{array}{c}
\dot{m}_{v} \\
\omega \\
q_{a}
\end{array}\right] \leq\left[\begin{array}{c}
0.015 \mathrm{~kg} \mathrm{~s}^{-1} \\
75 \mathrm{~Hz} \\
600 \mathrm{~W}
\end{array}\right]
$$

\section{Nonlinear Simulation Results}

\subsection{Fixed Horizon Optimization}

Before solving the receding horizon optimization in MPC, we first consider fixed horizon optimal control based on the linearized system applied to the nonlinear simulation. The goal is to tune the optimization horizon and various weighting parameters before implementing on our testbed.

Consider a steady $q_{e}^{*}=1000 \mathrm{~W}$ heat load. The nominal control to maximize the COP and $\mathrm{CHF}$ results in [9]:

$$
\begin{aligned}
& u^{*}=\left[\begin{array}{llll}
0.0076 \mathrm{~kg} \mathrm{~s}^{-1} & 50 \mathrm{~Hz} & 24 \mathrm{~W}
\end{array}\right]^{T}, \quad A_{v}^{*}=28 \% . \\
& x^{*}=\left[\begin{array}{llllllll}
0.65 & 700 \mathrm{kPa} & 21{ }^{\circ} \mathrm{C} & 0.55 & 170 \mathrm{kPa} & -8.4{ }^{\circ} \mathrm{C} & 18{ }^{\circ} \mathrm{C} & -7.6{ }^{\circ} \mathrm{C}
\end{array}\right]^{T} \text {. }
\end{aligned}
$$

Fig. 4 compares the optimal control under $300 \mathrm{~W} 800 \mathrm{~s}$ heat pulse with different pulse lead times, $600 \mathrm{~s}$ vs. $1500 \mathrm{~s}$, and optimization horizons, $1500 \mathrm{~s}$ vs. $2500 \mathrm{~s}$ (solid vs. dash lines in the figure). The optimal control strategy shows the following trends:

a. From the steady state energy balance, the heat load $q_{e}$ is proportional to the mass flow rate $\dot{m}_{v}$. So to remove the increased heat load, $\dot{m}_{v}$ needs to build up to a sufficiently high level. 
b. The CHF increases with $P_{e}$ and decreases with $\dot{m}_{v}$ (see (5)). Since $\dot{m}_{v}$ is already high during disturbance (from (a)), $P_{e}$ needs to be sufficiently high to ensure the higher heat flux does not violate the CHF.

c. Increasing $q_{a}, \omega$, and $A_{v}$ will all contribute to higher $\dot{m}_{v}$. However, higher $\omega$ would lower $P_{e}$ (see (4)) and higher $A_{v}$ would lower $P_{c}$ (see (3)), possibly violating the minimum $P_{c}$ constraint. Therefore, $q_{a}$ is needed to supply the required higher demand on the mass flow rate.

d. The compressor speed $\omega$ decreases to increase $P_{e}$ and hence the CHF.

e. When $q_{a}$ is able to supply sufficient mass flow, $A_{v}$ can decrease to build up the pressure difference $P_{c}-P_{e}$ that can be released during disturbance as additional mass flow rate.

The accumulator heat saturates at its upper bound initially in anticipation of the incoming disturbance, which deploys extra refrigerant from the accumulator tank. Since the optimization knows the pulse is of finite length, $q_{a}$ starts to decrease before the arrival of the heat pulse, after enough refrigerant has already been deployed for the predicted heat pulse. Though the accumulator heat $q_{a}$ can increase the mass flow rate by boiling off refrigerant in the tank, its effect is slower than the valve and compressor due to the large thermal storage in the accumulator wall, band heaters and the large accumulator volume.

In the short lead time case, the control action is more aggressive (see the large jump of the control effort right at the start), and acceptable temper-

ature is maintained during the heat pulse. In the long lead time case, the control action is in the same direction but less aggressive. However, the accumulation of the linearization error over the longer period results in the linear model predicting, erroneously, that CHF is avoided. Hence, the control effort based on the linear model is inadequate to prevent $\mathrm{CHF}$ violation in the nonlinear model. This points out the importance of applying measurement feedback, as in model predictive control, to correct for the modeling error due to linearization.

\subsection{Receding Horizon Optimization: Effect of Disturbance Pulse Magnitude and Prediction Horizon}

The receding horizon optimization in MPC is based on the fixed horizon optimization (same control objectives and constraints) but with the initial state updated by measurements. The controller only implements a fixed number of steps of the optimal control and then re-performs the optimization with the new initial condition. The fixed horizon optimization includes 

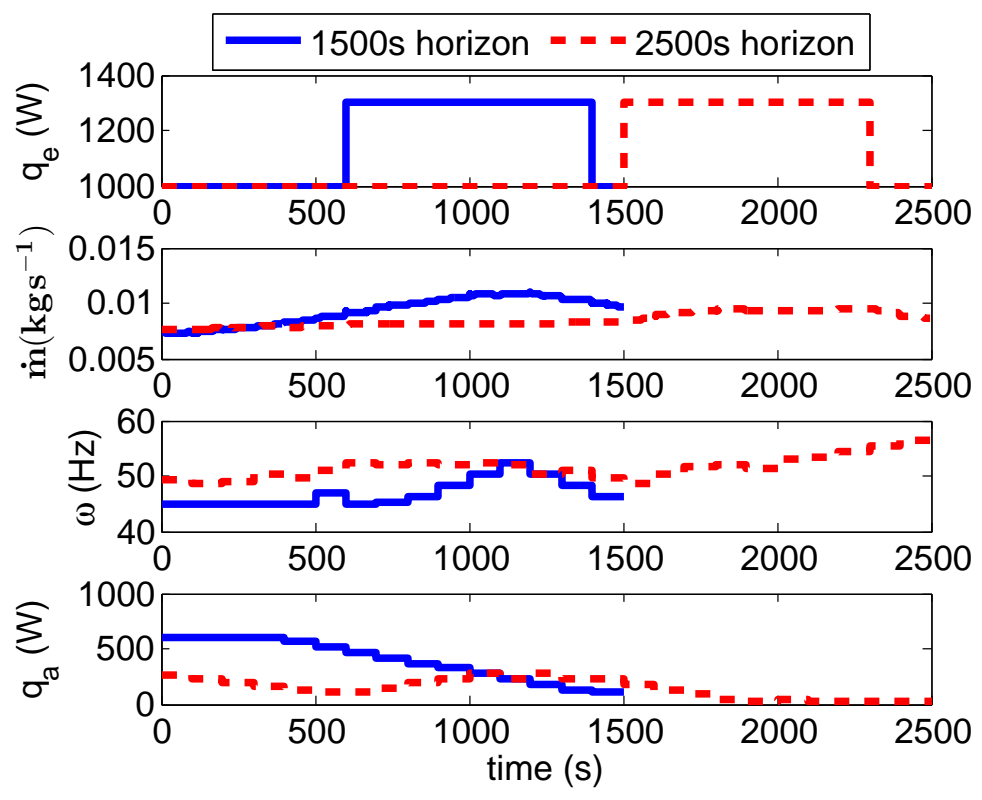

(a) Inputs

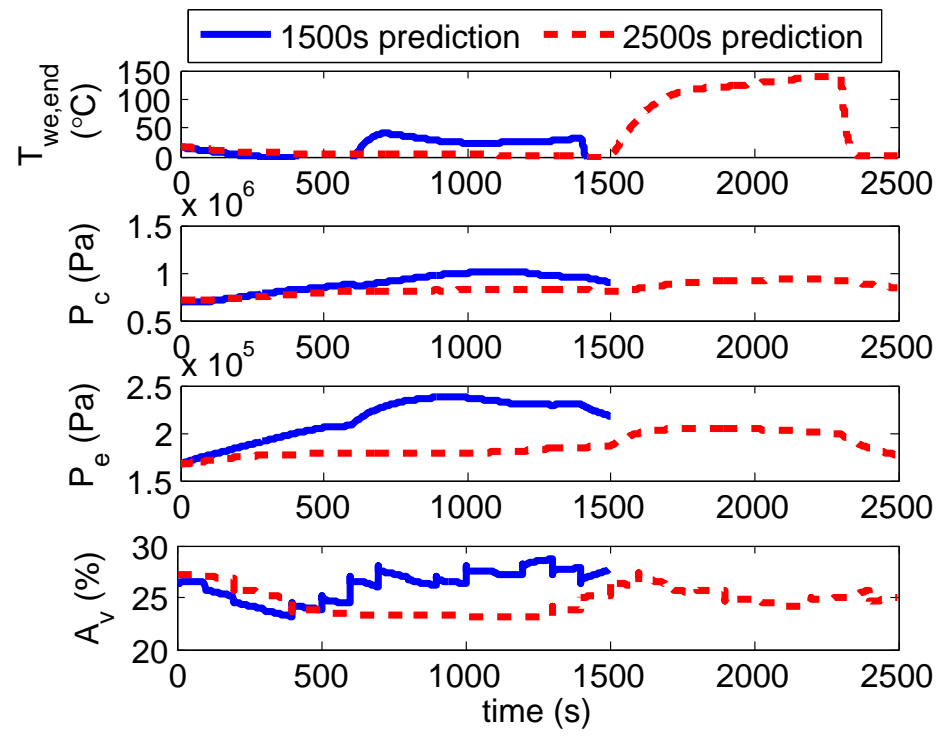

(b) Outputs

Fig. 4: Nonlinear simulation of open-loop optimal control and comparison of different optimization horizon. Longer prediction horizons cause false prediction of CHF due to model-mismatch accumulation (see the dashed red line in Plot 1 of Fig. 4(b)). (control interval $=100 \mathrm{~s})$ 
the entire disturbance profile in its optimization horizon, and therefore starts to prepare for the incoming disturbance right at $t=0$. With the receding horizon, MPC does not start to prepare for the incoming disturbance until the disturbance appears inside the prediction horizon. The time constraint of the online optimization requires the MPC to have a shorter optimization horizon than the open-loop optimal control. The measurement-based update of the initial state also enables the MPC to constantly correct for the errors between system and model dynamics. The fixed horizon optimization result in the previous section suggests that the prediction horizon in MPC should not be too long, as significant linearization error could accumulate and affect the validity of the optimization result. In this section, we focus on the effect of the length of the prediction horizon, which determines the lead time of a disturbance, defined as the time between the first emergence of the disturbance in the optimization horizon and its actual arrival. During the lead time, the MPC can move the system to a more advantageous operating point in preparation for the arrival of the disturbance.

We consider three pulse power levels, $100 \mathrm{~W}, 300 \mathrm{~W}$, and $500 \mathrm{~W}$ to evaluate the corresponding required lead time. For the $100 \mathrm{~W}$ pulse, the faster actuators such as the valve and compressor can quickly provide sufficient additional refrigerant, so a short lead time of $100 \mathrm{~s}$, or $N_{p}=2$ (each control step is $50 \mathrm{~s}$ ), is sufficient to prevent evaporator wall overheating. At $300 \mathrm{~W}$, $N_{p}=2$ is not sufficient to prevent CHF violation. For such a short horizon, accumulator heat has little effect due to its slow dynamics. Increasing the refrigerant mass flow using the compressor would reduce the evaporator pressure and in turn cause a drop in the critical quality (lower CHF). When the prediction horizon is increased to $500 \mathrm{~s}$, or $N_{p}=10$, the accumulator heat has sufficient time to add extra refrigerant into circulation. As a result, the MPC is able to avoid the excessive wall temperature rise. At $500 \mathrm{~W}$ and $100 \mathrm{~s}$ duration, we are not able to find a lead time to prevent CHF violation. The refrigerant mass flow rate is not able to go beyond $0.012 \mathrm{~kg} \mathrm{~s}^{-1}$, due to the saturation of inputs, $q_{a}$ at its upper bound, and $\omega$ at its lower bound. In this case, the MPC has reached its limit due to the physical bounds and constraints of the VCC. Since $500 \mathrm{~W}$ heat pulse lasting for $100 \mathrm{~s}$ will cause CHF regardless of lead time, heat pulses over $500 \mathrm{~W}$ with long duration is harmful to the current VCC testbed setup even with closed-loop control. Based on the nonlinear simulation, we conclude that pulses close to $100 \mathrm{~W}$ require a lead time of $\sim 100 \mathrm{~s}$; pulses of $300 \mathrm{~W}$ requires a lead time of $\sim 500 \mathrm{~s}$; pulses around $400 \mathrm{~W}$ requires a lead time of $\sim 1000 \mathrm{~s}$. Pulses above $500 \mathrm{~W}$ would be 
harmful to the system and should be avoided. This limitation is due to the capacity of the cooling system. In general, MPC relies more on the fast actuators to increase the mass flow rate for disturbances with short lead time. For longer lead times (greater than $100 \mathrm{~s}$ ), accumulator heat principally supplies the refrigerant for the increased mass flow requirement.

\section{Experimental Results}

\subsection{MPC for a Single Disturbance Heat Pulse}

In this section, we present the experimental implementation of the MPC controller for transient heat pulse rejection. The same nominal operating point is chosen as in Section 4. The nonlinear simulation results help to guide the selection of the prediction horizon for a given heat pulse. We first consider a $300 \mathrm{~W} 800$ s heat load pulse. This represents a sizable disturbance for the chosen operating point (the distance to the critical quality is only $\Delta x_{\text {crit }}=x_{\text {crit }}-x_{e, o}=0.02$ ). Indeed, experimental results in [10] showed that a well tuned gain-scheduling controller without disturbance prediction can only avoid a $250 \mathrm{~W}$ disturbance from this and similar operating points. Based on the discussion before, we choose $N_{p}=10$ (at a control interval of $50 \mathrm{~s}$ ) for the MPC implementation. Fig. 5 shows the experimental results from our testbed, with the heat pulse arriving at $t=800 \mathrm{~s}$. The prediction horizon is $50 N_{p}=500 \mathrm{~s}$, therefore, the heat pulse first appears in the optimization at $t=300 \mathrm{~s}$. The actuators do not react to the heat pulse until the first round of optimization is finished at $t=350 \mathrm{~s}$. The controller then reacts aggressively to the incoming disturbance, similar to the simulation results. The evaporator inlet mass flow rate and the evaporator and condenser pressures all ramp up as soon as the heat pulse comes into view in the prediction horizon. To meet the cooling demand, the accumulator heat input operates at its upper bound to add the maximum amount of refrigerant to the system. The compressor speed drops to its lower bound to ensure the evaporator pressure is sustained. The valve opening initially decreases to build up pressure, and then opens during the heat pulse disturbance to increase the mass flow rate. When the end of the heat pulse starts to appear in the prediction horizon, the control starts to move back to the nominal values (this is most evident for $q_{a}$ ). When the heat pulse completely passes, all control actuators return to the original operating point. 


\subsection{Dynamic Prediction Horizon}

The prediction horizon should be long enough for the preparation of the incoming disturbance, and at the same time, as short as possible to prevent excessive computation. We propose to adjust the prediction horizon length based on the height and duration of the heat load. This may be stored in a look-up table based on simulation or experimental results. As an example, consider a $300 \mathrm{~W}$ pulse which strains the ability of a $500 \mathrm{~s}$ (10 control steps) prediction horizon to prepare, as seen from the saturation of the $q_{a}$ and $\omega$. When we increase the heat pulse to $400 \mathrm{~W}$, the $500 \mathrm{~s}$ horizon is insufficient to avoid CHF, as seen in the dashed line in plot 1 of Fig. 6(b). In this case, if the prediction horizon is doubled to $1000 \mathrm{~s}$ (last section shows that the $1000 \mathrm{~s}$ horizon is able to handle larger heat load pulses than the $500 \mathrm{~s}$ horizon.) The preparation now starts much earlier (shown in solid lines in Plots 2-4 of Fig. 6(a)) resulting in higher mass flow rate and heat exchanger pressures. As a result, large temperature rise is avoided. In general, the performance of the MPC is improved by extending the prediction horizon. If the disturbance is too large for the MPC to handle with 500 s prediction horizon (dashed red line in Plot 1 of Fig. 6(b)), it may be possible to extend the prediction horizon to achieve better performance (solid blue line in Plot 1 of Fig. 6(b)). However, one needs to keep in mind the two costs associated

with this extension of prediction horizon: the increase in optimization time and the increased model mismatch at the end of the prediction horizon.

\subsection{Effect of Pulse Shape}

Due to different applications, cooling systems encounter various types of transient heat pulses. For example, the transient heat disturbances encountered by a server blade during peak and off-peak operations are long but relatively small in power; the transient heat disturbance for discharging electromagnetic weaponry is short but much larger in power. Disturbance pulses may also follow each other and form a pulse train, such as during the continuous firing of an electromagnetic weapon. In this section, we investigate the effect of heat pulse types, varying power and duration, on the performance of MPC.

Consider three types of heat pulses shown in the top plot of Fig. 7 .

(a) $200 \mathrm{~W}$ pulse over $300 \mathrm{~s}$

(b) $200 \mathrm{~W}$ pulse over $50 \mathrm{~s}$

(c) $100 \mathrm{~W}$ pulse over $600 \mathrm{~s}$ 

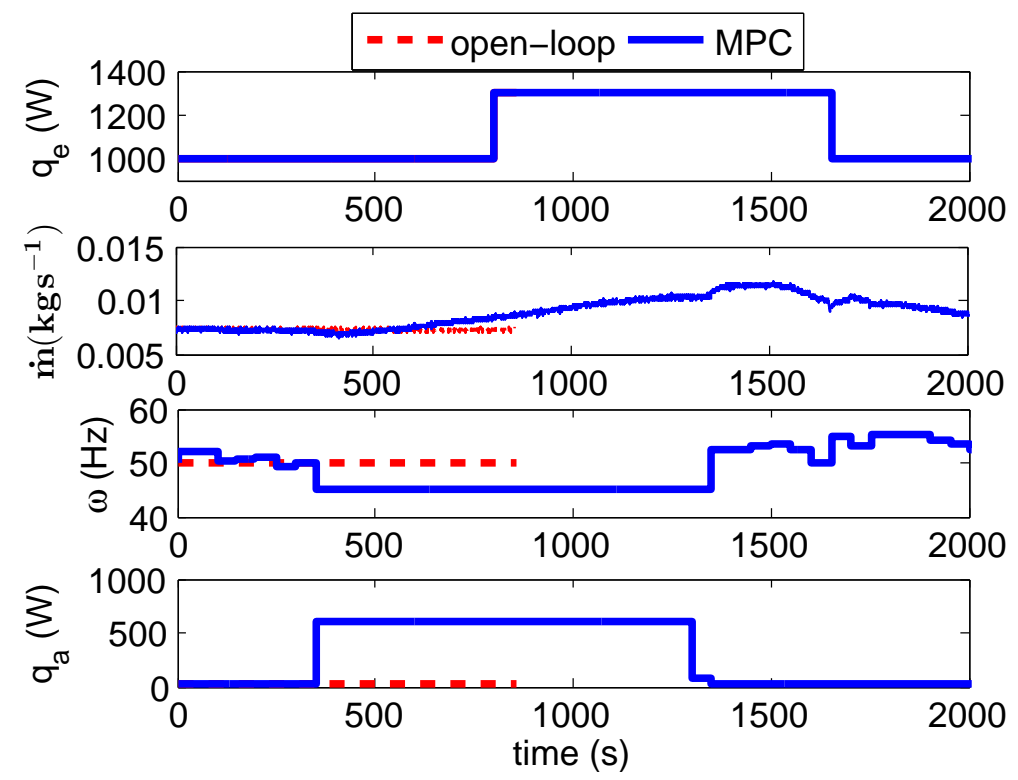

(a) Inputs

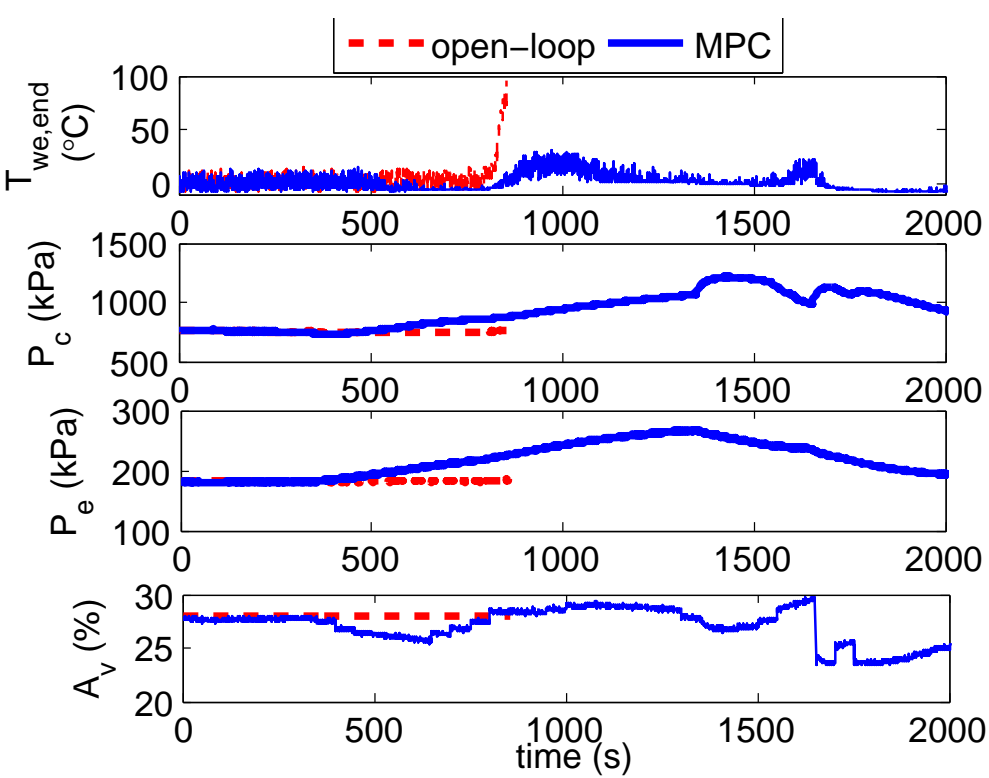

(b) Outputs

Fig. 5: Experimental comparison of MPC vs. open loop operation on predicted $300 \mathrm{~W}$ $800 \mathrm{~s}$ heat pulse, open-loop reaches CHF , and MPC successfully avoids CHF by deploying additional refrigerant mass flow (solid blue line in Plot 2 of Fig. 5(a)) and elevating the evaporator pressure (solid blue line in Plot 3 of Fig. 5(b)). 

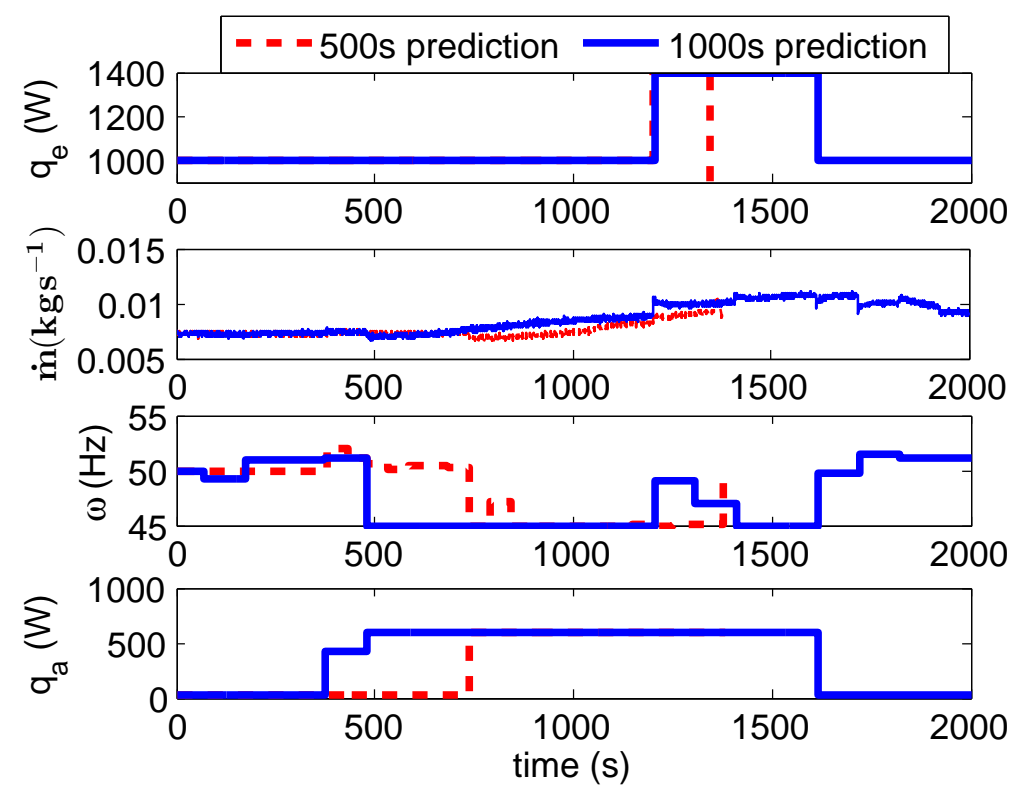

(a) Inputs
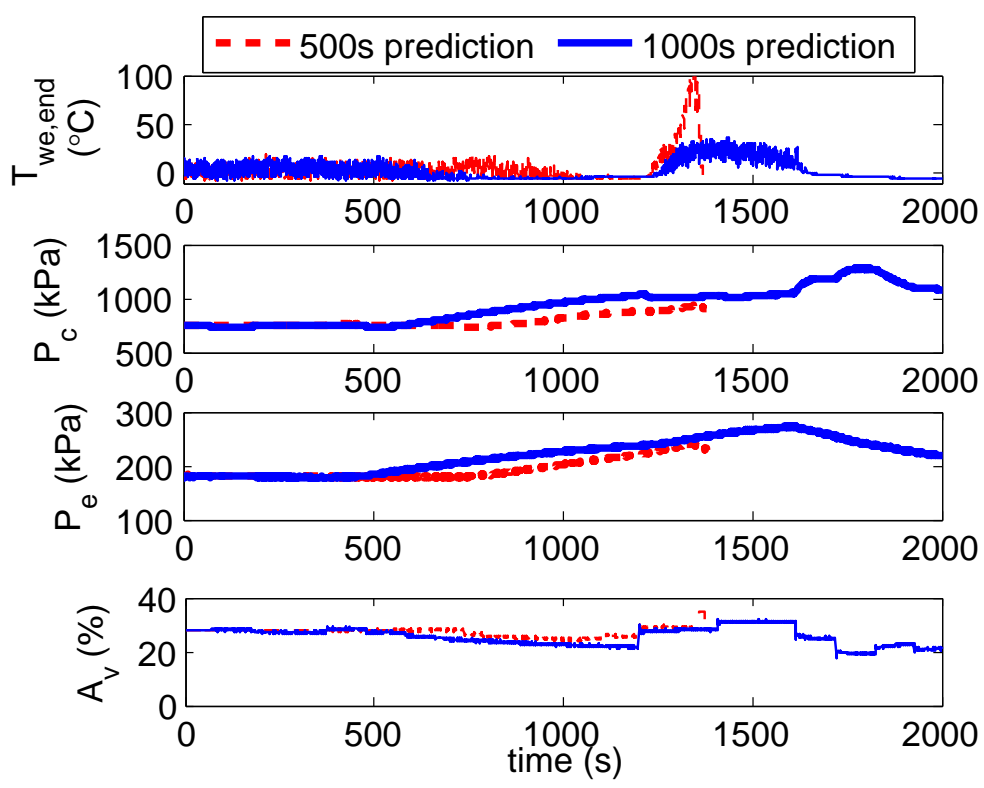

(b) Outputs

Fig. 6: Experimental comparison between $500 \mathrm{~s}$ and $1000 \mathrm{~s}$ prediction horizons on a $400 \mathrm{~W}$ pulse, $500 \mathrm{~s}$ prediction (control interval $=50 \mathrm{~s}$ ) is not long enough to reject the disturbance dashed red line in Plot 1 of Fig. 6(b) shows CHF using MPC with $500 \mathrm{~s}$ prediction horizon); CHF is successfully rejected by extending the disturbance prediction to $1000 \mathrm{~s}$ (control interval $=100 \mathrm{~s})$ (solid blue line in Plot 1 of Fig. 6(b)) 
The first two have the same magnitude but differ in duration. The third pulse has a smaller magnitude but longer duration; the total energy imparted on the cooling system is the same as the first case.

MPC with 500 s prediction horizon successfully handled all three cases as shown in Fig. 7. The preparation phase for (a) and (b) are similar with rapid ramp up of the mass flow rate and the heat exchanger pressures. To supply the mass flow, the accumulator heat reaches its upper bound. The compressor lowers its speed to the minimum and the valve reduces its opening to build up pressure in the preparation phase, and release the pressure to increase the mass flow during the heat pulse. The main difference between the long and short pulses is that the control effort returns to the nominal much faster for the short pulse case as the duration of the pulse is fully within the prediction horizon. For case (c), the system behaves similarly, except that the control effort is less aggressive (due to the control weighting) and does not reach its constraint boundaries. To reduce energy consumption, the MPC relies more on the valve and compressor to build up the mass flow rate and does not saturate the accumulator heat. The control duration is longer due to the longer disturbance pulse.

For repeated operations, the disturbance may consist of a pulse train. If the pulses are far from each other (much longer than the prediction horizon), MPC would treat each pulse individually (if the system has time to return to its nominal state). If the pulses are closer together, MPC would prepare for the multiple pulses together as a group similar as for a single longer pulse. Fig. 8 shows the MPC and open loop operation results under three $400 \mathrm{~W} 50 \mathrm{~s}$ pulses as in the top plot. The open-loop operation approaches CHF immediately after the arrival of the first pulse. With MPC at $500 \mathrm{~s}$ prediction horizon, CHF is successfully avoided. The results show a similar preparation stage as before - ramping up of the mass flow rate and pressures achieved by increased accumulator heat input, lower compressor speed and valve opening, and the release of the pressure during the heat load. As more of the heat pulse come into the prediction horizon, the control action, most notably the mass flow rate, becomes more aggressive. As pulses start to disappear from the prediction horizon, the control effort gradually returns to the nominal condition.

Pulse trains are easier for MPC to handle than a single longer pulse with the same magnitude because the VCC uses the intervals between pulses to recover and prepare for the next pulse. For example, the MPC with $500 \mathrm{~s}$ prediction horizon can reject three consecutive $400 \mathrm{~W}$ heat pulses with $50 \%$ 


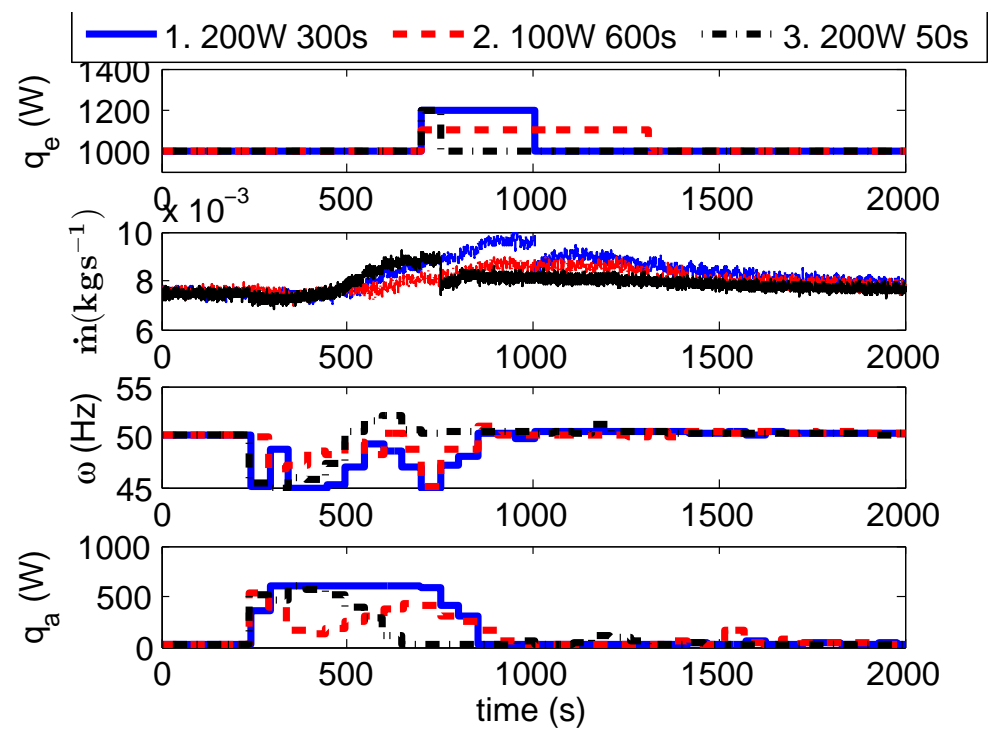

(a) Inputs

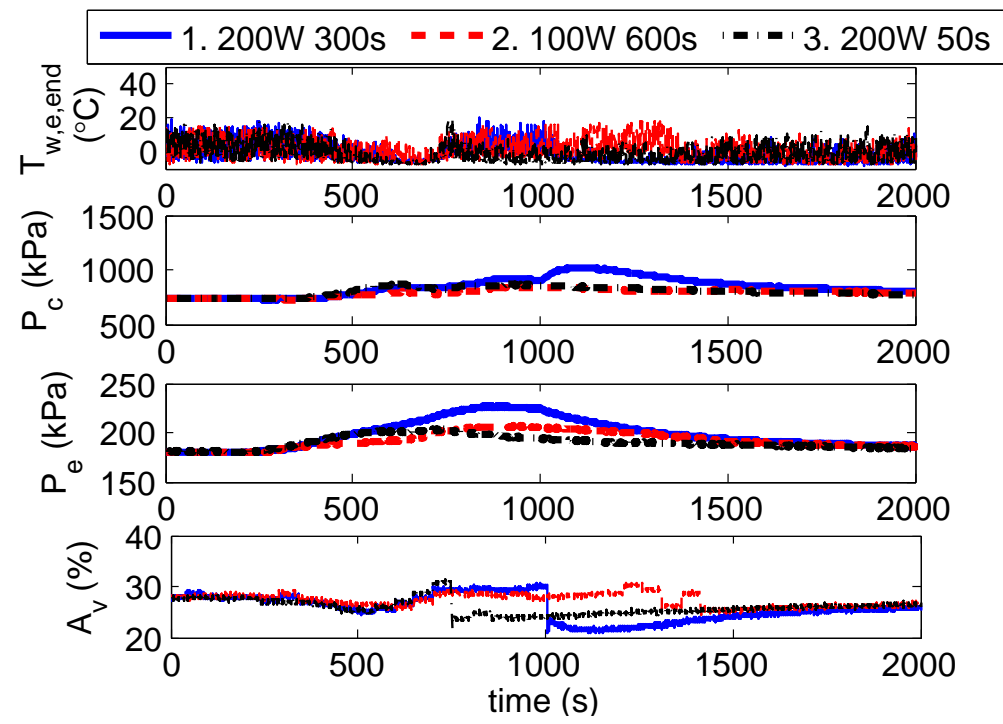

(b) Outputs

Fig. 7: Experimental comparison of disturbance rejection of various pulse types. MPC can relatively easily handle heat pulses with either high power or long duration. The pulse with both high power and long duration requires the most amount of cooling (largest mass flow rate deployed during disturbance as shown by the solid blue line in Plot 2 of Fig. 7(a)). 
duty cycle (solid lines in Fig. 8), however it cannot reject a single longer pulse of $400 \mathrm{~W}$ (dash lines in Fig. 6).

\section{Conclusion and Future Work}

This paper presents the results of applying $\mathrm{MPC}$ for $\mathrm{CHF}$ avoidance in the presence of known transient heat disturbances. MPC is ideally suited for this application due to its ability to prepare for known future disturbances and to handle complex constraints. With the known disturbance, MPC can improve the cooling capability of the VCC prior to the arrival of the disturbance. We apply a two-loop structure with the slower outer-loop optimizing for the refrigerant mass flow rate setpoints, compressor speed and accumulator heat and the faster inner-loop tracking the mass flow rate setpoints. We use the solution of the finite-horizon optimal control to guide the selection of prediction horizon for the MPC. Long prediction horizon could lead to accumulation of modeling error, while short prediction horizon may not provide enough time to prepare for large heat disturbances. We have shown through nonlinear simulation and experimentation on our VCC testbed that MPC with prediction horizon matched with the size of the disturbance can effectively prepare the system to counteract large transient heat disturbances. We have included results for pulse disturbances of various shapes and sizes as well as a pulse train.

This work considers MPC about a fixed operating point. We are currently exploring extension to gain scheduling to address large excursion away from the operating point or switching between operating points. We are also working on the extension to multiple-evaporator VCC systems by applying distributed optimization rather than a centralized scheme as we do now.

\section{Acknowledgment}

We would like to thank the reviewers for providing valuable constructing comments. This work is supported primarily by the Office of Naval Research (ONR) Award N00014-13-1-0189 entitled "Systems Level Thermal Management for Multiple High Transient Heat Loads," and in part by the National Science Foundation Smart Lighting Engineering Research Center (EEC-0812056) and in part by the Center for Automation Technologies and Systems (CATS) under a block grant from the New York State Empire State Development Division of Science, Technology and Innovation (NYSTAR). 

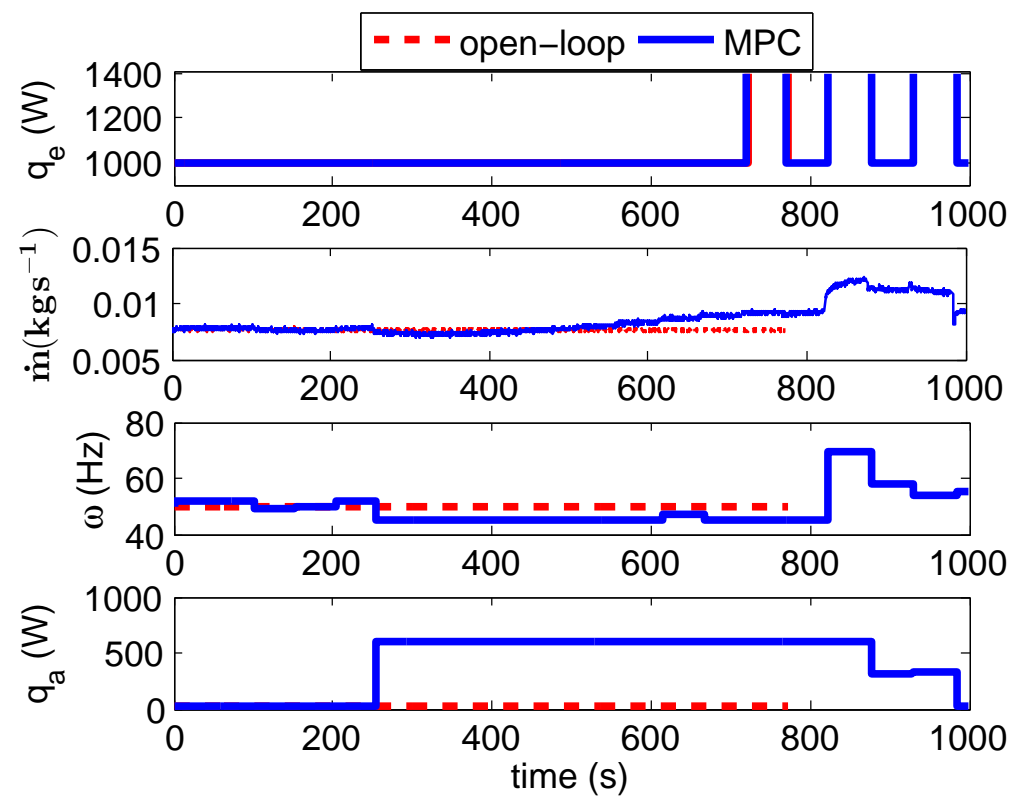

(a) Inputs
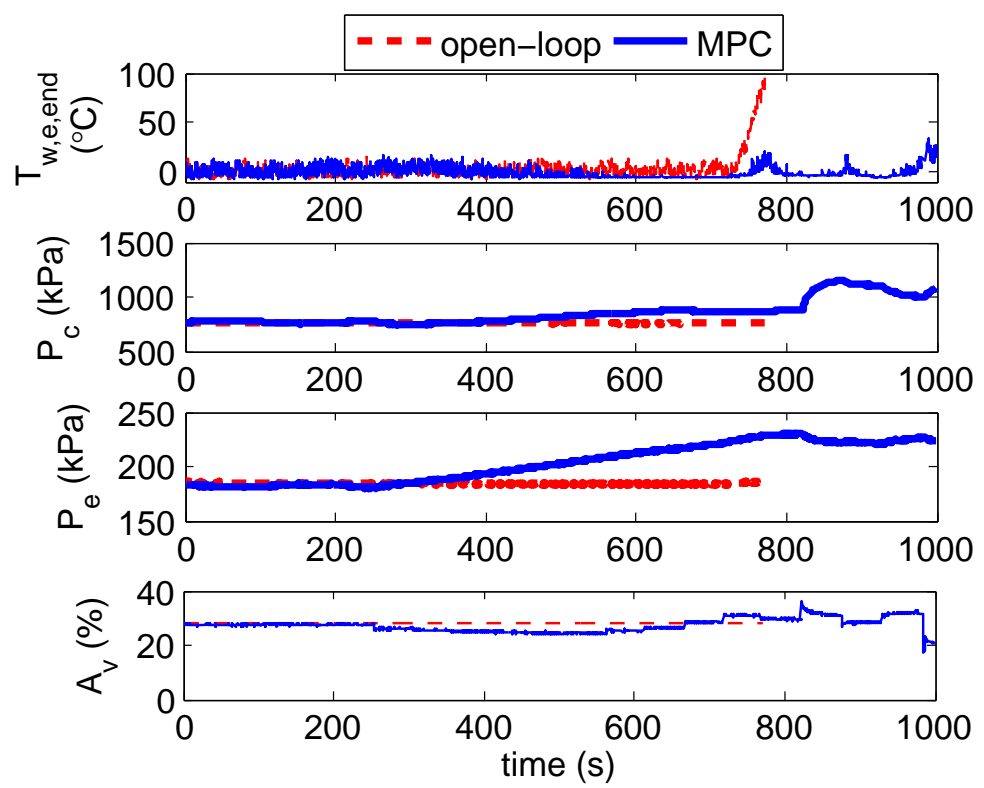

(b) Outputs

Fig. 8: Experimental comparison of pulse-train disturbance rejection with MPC vs. open loop operation on disturbance rejection of 3 consecutive pulses of $400 \mathrm{~W}$, open-loop operation reaches CHF (dashed red line in Plot 1 of Fig. 8(b)) and MPC successfully avoids CHF (solid blue line in Plot 1 of Fig. 8(b)). MPC prepares for these three pulses together (see Plot 4 of Fig. 8(a)) because the pulses are close to each other. 


\section{References}

[1] X.-D. He, S. Liu, H. H. Asada, and H. Itoh, "Multivariable control of vapor compression systems," HVACER Research, vol. 4, no. 3, pp. 205230, 1998.

[2] R. Shah, B. P Rasmussen, and A. G. Alleyne, "Application of a multivariable adaptive control strategy to automotive air conditioning systems," International Journal of Adaptive Control and Signal Processing, vol. 18, no. 2, pp. 199-221, Mar. 2004.

[3] B. P. Rasmussen and A. G. Alleyne, "Dynamic modeling and advanced control of air conditioning and refrigeration systems," Air Conditioning and Refrigeration Center. College of Engineering. University of Illinois at Urbana-Champaign., Tech. Rep., Jun. 2006.

[4] N. Jain, B. Li, M. Keir, B. Hencey, and A. Alleyne, "Decentralized feedback structures of a vapor compression cycle system," IEEE Transactions on Control Systems Technology, vol. 18, no. 1, pp. 185-193, Apr. 2009.

[5] J. B. Marcinichen, T. N. Holanda, and C. Melo, "A dual SISO controller for a vapor compression refrigeration system," in Refrig. Conf. at Purdue, West Lafayette-IN, USA, vol. 2444, 2008, pp. 1-8.

[6] J. B. Marcinichen, J. A. Olivier, and J. R. Thome, "On-chip two-phase cooling of datacenters: Cooling system and energy recovery evaluation," Applied Thermal Engineering, vol. 41, pp. 36-51, Aug. 2012.

[7] J. B. Marcinichen, J. R. Thome, and B. Michel, "Cooling of microprocessors with micro-evaporation: A novel two-phase cooling cycle," International Journal of Refrigeration, vol. 33, no. 7, pp. 1264-1276, Nov. 2010.

[8] J. B. Marcinichen and J. R. Thome, "Refrigerated cooling of microprocessors with micro-evaporation new novel two-phase cooling cycles: A green steady-state simulation code," in 13th Brazilian congress of thermal sciences and engineering. Uberlândia, MG, Brazil, 2010. 
[9] D. T. Pollock, Z. Yang, J. T. Wen, Y. Peles, and M. K. Jensen, "Modelbased control of vapor compression cycles for transient heat-flux removal," International Journal of Heat and Mass Transfer, vol. 77, pp. 662-683, Oct. 2014.

[10] Z. Yang, D. T. Pollock, and J. T. Wen, "Gain-scheduling control of vapor compression cycle for transient heat-flux removal," Control Engineering Practice, vol. 39, pp. 67-89, Jun. 2015.

[11] D. T. Pollock, Z. Yang, and J. T. Wen, "Dryout avoidance control for multi-evaporator vapor compression cycle cooling," Applied Energy, vol. 160, pp. 266-285, Dec. 2015.

[12] J. Catano, F. Lizarralde, T. Zhang, J. T. Wen, M. K. Jensen, and Y. Peles, "Vapor compression refrigeration cycle for electronics cooling-part II: gain-scheduling control for critical heat flux avoidance," International Journal of Heat and Mass Transfer, vol. 66, pp. 922-929, Nov. 2013.

[13] E. F. Camacho and C. B. Alba, Model predictive control. Springer Science \& Business Media, London, 2013.

[14] C. E. Garcia, D. M. Prett, and M. Morari, "Model predictive control: theory and practice - a survey," Automatica, vol. 25, no. 3, pp. 335-348, May 1989.

[15] M. S. Elliott, "Decentralized model predictive control of a multiple evaporator HVAC system," Master's thesis, Texas A\&M University, USA, 2008.

[16] T. G. Hovgaard, S. Boyd, L. F. Larsen, and J. B. Jørgensen, "Nonconvex model predictive control for commercial refrigeration," International Journal of Control, vol. 86, no. 8, pp. 1349-1366, Jan. 2013.

[17] L. F. S. Larsen, "Model based control of refrigeration systems," Ph.D. dissertation, Department of Control Engineering, Aalborg University, Danmark, 2006.

[18] A. Aswani, N. Master, J. Taneja, D. Culler, and C. Tomlin, "Reducing transient and steady state electricity consumption in hvac using learning-based model-predictive control," Proceedings of the IEEE, vol. 100, no. 1, pp. 240-253, 2012. 
[19] M. Wallace, B. Das, P. Mhaskar, J. House, and T. Salsbury, "Offsetfree model predictive control of a vapor compression cycle," Journal of Process Control, vol. 22, no. 7, pp. 1374-1386, Aug. 2012.

[20] M. Gräber, C. Kirches, J. P. Schlöder, and W. Tegethoff, "Nonlinear model predictive control of a vapor compression cycle based on first principle models," in MATHMOD, 7th Vienna International Conference on Mathematical Modelling, Vienna, Austria, 2012.

[21] R. Zhou, T. Zhang, J. Catano, J. T. Wen, G. J. Michna, Y. Peles, and M. K. Jensen, "The steady-state modeling and optimization of a refrigeration system for high heat flux removal," Applied Thermal Engineering, vol. 30 , no. 16 , pp. 2347-2356, Nov. 2010.

[22] AW Company, "ACM mass coriolis mass flow meter," http://datasheets.globalspec.com/ds/163/AWCompany/738BDA755A77-4155-BA04-B05126E02050, Online; accessed September 12, 2016.

[23] J. Catano, T. Zhang, J. T. Wen, M. K. Jensen, and Y. Peles, "Vapor compression refrigeration cycle for electronics cooling-part I: Dynamic modeling and experimental validation," International Journal of Heat and Mass Transfer, vol. 66, pp. 911-921, Nov. 2013.

[24] J. E. Catano, "Dynamic modeling and advanced control of vapor compression cycles for electronics cooling," Ph.D. dissertation, Rensselaer Polytechnic Institute, 2011.

[25] L. Levitan and F. Lantsman, "Critical heat fluxes in internally heated annular channels," Therm. Eng. (USSR) (Engl. Transl.); (United States), vol. 24:4, Jan 1978.

[26] M. S. Elliott and B. P. Rasmussen, "Model-based predictive control of a multi-evaporator vapor compression cooling cycle," in American Control Conference, 2008. IEEE, 2008, pp. 1463-1468.

[27] TRICOR, "Instruction Manual TRICOR Coriolis Mass Flow Meter," http://tricorflow.com/wp-content/uploads/2016/09/TCM _E80_M_EN_160520_E004, Online; accessed December, 2016. 
[28] T. Brunschwiler, B. Smith, E. Ruetsche, and B. Michel, "Toward zeroemission data centers through direct reuse of thermal energy," IBM Journal of Research and Development, vol. 53, no. 3, pp. 11:1-11:3, May 2009.

[29] Intel, "Intel ${ }^{\circledR}$ core $^{\mathrm{TM}} \mathrm{m}$ processor family datasheet volume 1 of $2, " 2014$. 